\title{
Measurements of presolar grains
}

\section{Peter Hoppe $^{1}$}

Max Planck Institute for Chemistry

J.-J. Becherweg 27, 55128 Mainz, Germany

E-mail: peter.hoppe@mpic.de

Primitive solar system materials contain nanometer- to micrometer-sized presolar grains that formed in the winds of evolved stars or in the ejecta of stellar explosions. Laboratory studies of individual presolar grains have provided a wealth of astrophysical information, such as on stellar nucleosynthesis and evolution, mixing in stars, Galactic chemical evolution, grain formation in stellar environments, chemical and physical processes in the ISM, and on the types of stars that contributed dust to the Solar System. Among the identified presolar minerals are silicon carbide (SiC), graphite, silicon nitride, refractory oxides, and silicates. The isotopic ratios of the major and minor elements in presolar grains range over many orders of magnitude, indicative of contributions from different stellar sources. Most presolar $\mathrm{SiC}$ (>90 \%) and oxide/silicate (ca. 90 $\%)$ grains apparently formed in the winds of low- to intermediate-mass AGB stars, as inferred mainly from heavy element isotopic compositions $(\mathrm{SiC})$ and, respectively, O-isotopic ratios (oxides/silicates). Although less abundant than grains from AGB stars, grains from Type II supernovae (SNe) are of particular importance. These grains incorporated matter from the outer $\mathrm{H}$-rich zone down to the innermost Ni-rich zone in variable proportions, as indicated by specific isotopic fingerprints. A couple of recent findings have advanced our understanding of SN grains, providing new insights into SN nucleosynthesis, chemistry, and dust formation.

11th Symposium on Nuclei in the Cosmos - NIC XI

Heidelberg, Germany

July 19-23 2010

\footnotetext{
$1 \quad$ Speaker
} 


\section{Introduction}

Dust is an important constituent of the interstellar medium (ISM). Dust forms around evolved stars and possibly also in the ISM. When our Sun and its planetary system formed by gravitational collapse of an interstellar cloud some 4.6 Gy ago, most of the contained interstellar dust was destroyed or heavily altered. Only a small fraction of it survived unaltered inside small planetary bodies, the asteroids and comets. Fragments from these bodies reach the Earth as meteorites and interplanetary dust particles (IDPs), in which pristine interstellar grains can be identified by their anomalous isotopic compositions. Because these grains are older than our solar system they have been named "presolar grains".

The first presolar grains, diamond and silicon carbide (SiC), were physically and chemically isolated from meteorites by Ed Anders and co-workers at the University of Chicago in the 1980s [1-2]. It became quickly clear that presolar SiC represents a sample of stardust. Whether also most of the diamonds have a stellar origin is still not clear. This is because diamonds are only 2-3 nm in size, which prevents isotope studies of single grains; the C-isotopic composition of ensembles of grains, containing millions of diamonds, is essentially solar and pronounced isotope anomalies are seen only for minor elements (e.g., Xe and Te) which, however, may be carried only by a small fraction of the grains.

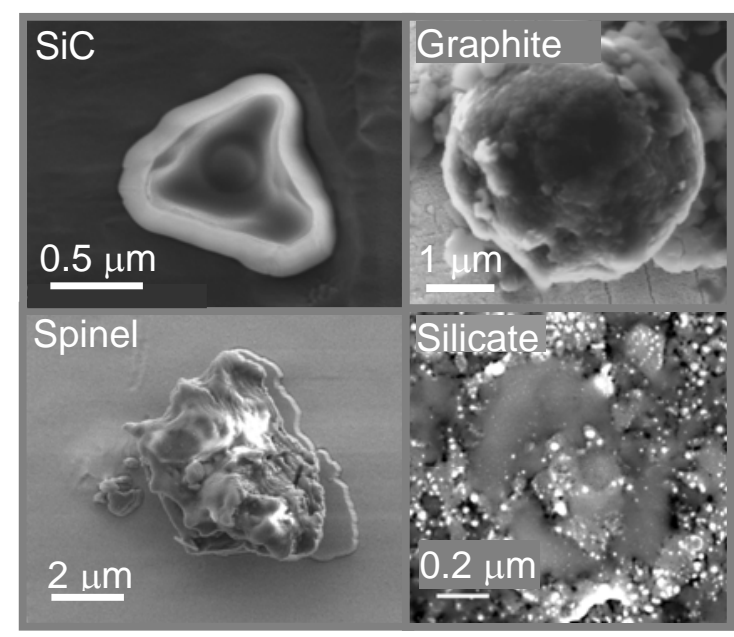

Figure 1. Pictures of presolar grains from primitive meteorites: $\mathrm{SiC}$ (upper left), graphite (upper right), spinel ( $\mathrm{MgAl}_{2} \mathrm{O}_{4}$, lower left), and a grain consisting of an Al-rich core surrounded by a silicate mantle (lower right). The shown $\mathrm{SiC}$ grain is from a supernova (SN), the graphite grain from an asymptotic giant branch (AGB) star or a SN, and the spinel and silicate grains are from AGB stars. Photo credit: Max Planck Institute for Chemistry.

Following the discovery of presolar diamond and $\mathrm{SiC}$ other presolar minerals have been found in the last two decades, namely, graphite, silicon nitride, refractory oxides (mainly $\mathrm{Al}_{2} \mathrm{O}_{3}$, $\mathrm{MgAl}_{2} \mathrm{O}_{4}$, and $\mathrm{CaAl}_{12} \mathrm{O}_{19}$ ), and a variety of silicates. Like SiC, all these presolar minerals have sizes $>100 \mathrm{~nm}$ and sometimes even $>1 \mu \mathrm{m}$ (Fig. 1 ). This permits single grain isotope studies and it was shown that all these presolar minerals are not only interstellar grains but must have formed in the winds of evolved stars or in the ejecta of stellar explosions (Table 1) [3-5].

Laboratory studies, especially by secondary ion mass spectrometry (SIMS), resonance ionization mass spectrometry (RIMS), and electron microscopy, have provided a wealth of astrophysical information, such as on stellar nucleosynthesis and evolution, mixing in stars, Galactic chemical evolution (GCE), grain formation in stellar environments, chemical and physical processes in the ISM, and on the types of stars that contributed dust to the solar system. Here, I will briefly review the current state of our knowledge on presolar grains (section 2), 
discuss several recent findings that have advanced our understanding of SN grains (section 3), and present some unsolved problems (section 4).

Table 1. Presolar minerals with stellar origins from primitive meteorites.

\begin{tabular}{|c|c|c|c|c|}
\hline Mineral & $\begin{array}{l}\text { Abund. } \\
\text { (ppm) }{ }^{1}\end{array}$ & Isotopic Signatures & Stellar Source & $\begin{array}{c}\text { Rel. } \\
\text { Contr. }\end{array}$ \\
\hline$\overline{\mathrm{SiC}}$ & 30 & $\begin{array}{c}\text { Enh. }{ }^{13} \mathrm{C},{ }^{14} \mathrm{~N} \text {; Ne-E }(\mathrm{H}){ }^{2} \text {; s-process elem. } \\
\text { Low }{ }^{12} \mathrm{C} /{ }^{13} \mathrm{C} \text {, often enhanced }{ }^{15} \mathrm{~N} \\
\text { Enh. }{ }^{15} \mathrm{~N},{ }^{28} \mathrm{Si} \text {; extinct }{ }^{26} \mathrm{Al},{ }^{44} \mathrm{Ti},{ }^{49} \mathrm{~V} \\
\text { Enhanced }{ }^{12} \mathrm{C},{ }^{29} \mathrm{Si},{ }^{30} \mathrm{Si} \\
\text { Low }{ }^{12} \mathrm{C} /{ }^{13} \mathrm{C},{ }^{14} \mathrm{~N} /{ }^{15} \mathrm{~N} \text {; enhanced }{ }^{30} \mathrm{Si}\end{array}$ & $\begin{array}{c}\text { AGB }\left(1.5-3 \mathrm{M}_{\odot}\right) \\
\text { J-type C stars, post-AGB } \\
\text { SNII } \\
\text { SNII } \\
\text { Novae }\end{array}$ & $\begin{array}{c}>90 \% \\
<5 \% \\
1 \% \\
0.3 \% \\
0.1 \%\end{array}$ \\
\hline Graphite & 10 & $\begin{array}{c}\text { Enh. }{ }^{15} \mathrm{~N},{ }^{18} \mathrm{O},{ }^{28} \mathrm{Si} \text {; ext. }{ }^{26} \mathrm{Al},{ }^{41} \mathrm{Ca},{ }^{44} \mathrm{Ti},{ }^{49} \mathrm{~V} \\
\text { s-process elem.; subgrain composition } \\
\text { Low }{ }^{12} \mathrm{C} /{ }^{13} \mathrm{C} \\
\text { Low }{ }^{12} \mathrm{C} /{ }^{13} \mathrm{C} \text {; enhanced }{ }^{30} \mathrm{Si} \text {; Ne-E }(\mathrm{L}){ }^{2}\end{array}$ & $\begin{array}{c}\text { SNII } \\
\text { AGB } \\
\text { J-type C stars, post-AGB } \\
\text { Novae } \\
\end{array}$ & $\begin{array}{c}\sim 60 \% \\
\sim 30 \% \\
<10 \% \\
2 \%\end{array}$ \\
\hline$\overline{\mathrm{Si}_{3} \mathrm{~N}_{4}}$ & 0.002 & Enhanced ${ }^{15} \mathrm{~N},{ }^{28} \mathrm{Si}$; extinct ${ }^{26} \mathrm{Al}$ & $\overline{\text { SNII }}$ & $100 \%$ \\
\hline $\begin{array}{l}\text { Oxides/ } \\
\text { silicates }\end{array}$ & $\begin{array}{c}50 / \\
200^{3}\end{array}$ & $\begin{array}{c}\text { Enhanced }{ }^{17} \mathrm{O} \text {, depleted or } \sim \text { solar }{ }^{18} \mathrm{O} \\
\text { Enhanced }{ }^{17} \mathrm{O} \text {, strongly depleted }{ }^{18} \mathrm{O} \\
\text { Slightly enhanced }{ }^{16} \mathrm{O} \\
\text { Enhanced }{ }^{16} \mathrm{O} \text { or }{ }^{18} \mathrm{O} \text {; extinct }{ }^{44} \mathrm{Ti} \\
\text { High }{ }^{17} \mathrm{O} /{ }^{16} \mathrm{O} \text {, lower than solar }{ }^{18} \mathrm{O} /{ }^{16} \mathrm{O}\end{array}$ & $\begin{array}{c}\text { AGB }\left(1-2.2 \mathrm{M}_{\odot}\right) \\
\text { AGB }\left(<1.8 \mathrm{M}_{\odot}, \stackrel{\mathrm{CBP}}{ }\right) \\
\text { AGB (low } \mathrm{M} \& \mathrm{Z}), \mathrm{SNII} \\
\text { SNII } \\
\text { Novae }\end{array}$ & $\begin{array}{c}70 \% \\
15 \% \\
5 \% \\
10 \% \\
<1 \%\end{array}$ \\
\hline
\end{tabular}

1) Reported maximum values (normalized to volume of meteorite matrix).

$\left.{ }^{2}\right)$ Ne-E: Almost monoisotopic ${ }^{22} \mathrm{Ne}$ that is released at low (L) and high (H) temperatures, respectively;

$\mathrm{Ne}-\mathrm{E}(\mathrm{L})$ is probably from the decay of short-lived ${ }^{22} \mathrm{Na}$ (half-life $2.6 \mathrm{yr}$ ).

${ }^{3)}$ Higher abundances are observed in isotopically primitive IDPs.

\section{Isotopic compositions and stellar sources}

The most important isotopic signatures and the stellar sources of presolar minerals are summarized in Table 1. In the follwing I will focus on three types of presolar grains, namely, SiC, oxides, and silicates.

\subsection{Silicon carbide}

Based on the C-, N-, and Si-isotopic compositions, presolar SiC can be divided into distinct populations (Fig. 2) [6]. Most abundant are the mainstream (MS) grains which make up about $90 \%$ of all $\mathrm{SiC}$ grains. These grains have predominantly lower than solar ${ }^{12} \mathrm{C} /{ }^{13} \mathrm{C}$ and higher than solar ${ }^{14} \mathrm{~N} /{ }^{15} \mathrm{~N}$. They carry radiogenic ${ }^{26} \mathrm{Mg}$ from the decay of radioactive ${ }^{26} \mathrm{Al}$ (half life $700000 \mathrm{y}$ ); inferred ${ }^{26} \mathrm{Al} /{ }^{27} \mathrm{Al}$ ratios are typically between $10^{-4}$ and $10^{-3}$. The intermediatemass and heavy elements exhibit the signature of the s-process, pointing to 1.5-3 $\mathrm{M}_{\odot}$ AGB stars of about solar metallicity as stellar sources [7]. The detection of radioactive ${ }^{99}$ Tc (half life 200000 y) in the spectra of giant stars was the first direct observational evidence that the chemical elements are produced in the interior of stars [8]. Particularly interesting in this respect is the finding of radiogenic ${ }^{99} \mathrm{Ru}$ from the decay of ${ }^{99} \mathrm{Tc}$ in SiC MS grains [9]. Silicon in MS grains shows only small isotope anomalies $(<20 \%)$. Although $\mathrm{Si}$ is affected by the s-process in solar metallicity AGB stars, the dredge-up of s-process $\mathrm{Si}$ is expected to change the envelope Siisotopic composition only marginally. The observed Si-isotopic systematics of the MS grains (slope 1.37 line in a $\delta^{29} \mathrm{Si}$ vs. $\delta^{30} \mathrm{Si}$ plot, Fig. 3) must thus represent a range of starting compositions of the parent stars established by the GCE of the Si isotopes [10-12]. 
The $\mathrm{Y}$ and $\mathrm{Z}$ grains plot to the right of the $\mathrm{SiC}$ MS line, the signature of s-process $\mathrm{Si}$ dredge-up. These grains are believed to come from low- and intermediate mass AGB stars with $1 / 3$ to $1 / 2$ times solar metallicity [13-14]. SiC grains with ${ }^{12} \mathrm{C} /{ }^{13} \mathrm{C}$ ratios of $<10$, a large range of $\mathrm{N}$-isotopic ratios, and ${ }^{26} \mathrm{Al} /{ }^{27} \mathrm{Al}$ ratios somewhat higher than in MS grains make up the $\mathrm{A}$ and $\mathrm{B}$ grains [15]. Among the proposed stellar sources are J-type C stars and born-again AGB stars.

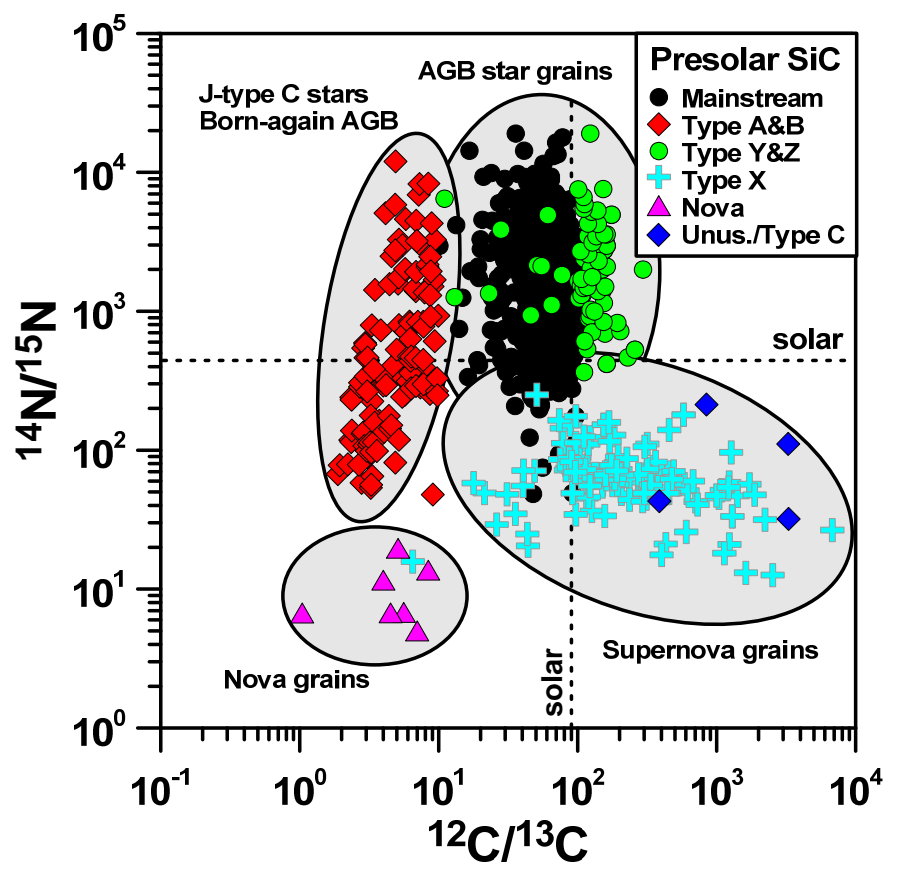

Figure 2. Carbon- and N-isotopic compositions of the different presolar $\mathrm{SiC}$ populations. The solar ratios are indicated by the dashed lines. Data sources: [16-19] for the unusual and Type $\mathrm{C}$ grains; for the other grains see [3-5].

$\mathrm{SiC}$ grains from $\mathrm{SNe}$ are the $\mathrm{X}$ grains [20-23]. These grains are characterized by enhanced ${ }^{12} \mathrm{C}$ (most grains) and ${ }^{15} \mathrm{~N}$, and lower than solar ${ }^{29} \mathrm{Si} /{ }^{28} \mathrm{Si}$ and ${ }^{30} \mathrm{Si} /{ }^{28} \mathrm{Si}$ ratios (Fig. 3). They have very high ${ }^{26} \mathrm{Al} /{ }^{27} \mathrm{Al}$ ratios of up to nearly 1 and they carry radiogenic ${ }^{44} \mathrm{Ca}$ and ${ }^{49} \mathrm{Ti}$ from the decay of radioactive ${ }^{44} \mathrm{Ti}$ (half life $60 \mathrm{y}$ ) and ${ }^{49} \mathrm{~V}$ (half life $330 \mathrm{~d}$ ). These isotopic signatures require deep and heterogeneous mixing in SNII ejecta. Some of the X grains exhibit an unusual Mo isotopic pattern (enrichments in ${ }^{95} \mathrm{Mo}$ and ${ }^{97} \mathrm{Mo}$ ) that can be explained by a neutron burst in shocked He-rich matter [24]. Related to the X grains are the presolar silicon nitride grains, all of which apparently come from SNe [25]. A new type of SiC grains from SNeII with isotopically heavy $\mathrm{Si}$ was recently discovered and will be discussed in section 3.

Very rare are $\mathrm{SiC}$ grains from novae which have very low ${ }^{12} \mathrm{C} /{ }^{13} \mathrm{C}$ and ${ }^{14} \mathrm{~N} /{ }^{15} \mathrm{~N}$ ratios (Fig. 2) as well as enhanced ${ }^{30} \mathrm{Si}$ (Fig. 3) [26]. It should be noted that not all grains with low ${ }^{12} \mathrm{C} /{ }^{13} \mathrm{C}$ and ${ }^{14} \mathrm{~N} /{ }^{15} \mathrm{~N}$ are from novae since also $\mathrm{SN}$ grains with this signature have been found [27].

\subsection{Oxides and silicates}

An important finding in the last decade was the discovery of presolar silicates. These grains remained unrecognised for a long time although, as it finally turned out, they are the most abundant presolar mineral if diamonds are ignored (Table 1). The reason for this is that they, contrary to carbonaceous presolar grains and the refractory presolar oxides, cannot be separated chemically from meteorites and only high spatial resolution isotope mapping of thin sections of meteorites and IDPs with the NanoSIMS made their discovery possible [28]. 


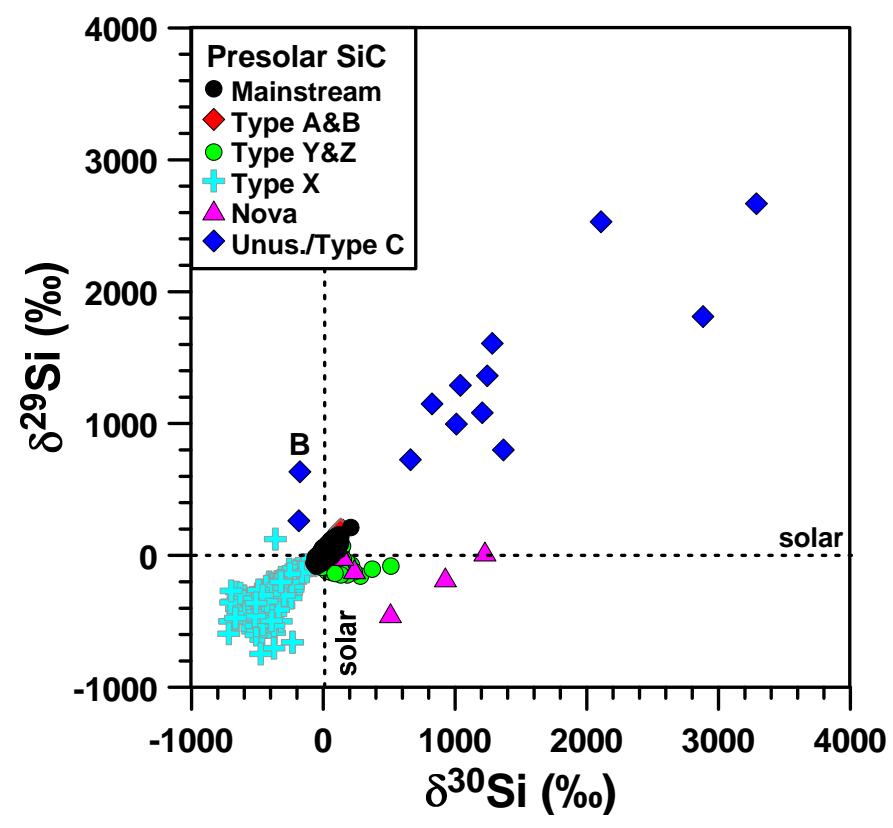

Figure 3. Silicon-isotopic compositions of the different presolar SiC populations given in permil deviation from the solar ratios. $\delta^{\mathrm{i}} \mathrm{Si}=$ $\left[\left({ }^{\mathrm{i}} \mathrm{Si} /{ }^{28} \mathrm{Si}\right) /\left({ }^{\mathrm{i}} \mathrm{Si} /{ }^{28} \mathrm{Si}\right)_{\odot}-1\right] \times 1000$. The solar ratios are indicated by the dashed lines. Data sources: [16-19, 29-30] for the unusual and Type $C$ grains; for the other grains see [3-5].

A large data set exists on the O-isotopic compositions of presolar oxides and silicates (Fig. 4). Similar to SiC, presolar O-rich dust is divided into distinct groups [31-32]. Most abundant are the Group 1 grains, which comprise about $70 \%$ of all grains. These grains show moderate to large enrichments in ${ }^{17} \mathrm{O}$ and close to solar or slightly lower than solar ${ }^{18} \mathrm{O} /{ }^{16} \mathrm{O}$. The most likely stellar sources are 1.2-2.2 $\mathrm{M}_{\odot}$ AGB stars of about solar metallicity. Because in red giant stars the ${ }^{17} \mathrm{O} /{ }^{16} \mathrm{O}$ ratio depends mainly on stellar mass, its distribution in AGB star grains is a sensitive measure of the mass distribution of the parent stars. The upper mass limit of $2.2 \mathrm{M}_{\odot}$ has been inferred from a comparison of the grain data with the results from a Monte Carlo simulation in which a Salpeter IMF was assumed [33]. The Group 2 grains ( 15\% of all grains) are strongly depleted in ${ }^{18} \mathrm{O}$ and moderately enriched in ${ }^{17} \mathrm{O}$. These grains are likely to originate from low-mass AGB stars in which cool bottom processing (CBP) [34] was operating. An alternative explanation is given by [35] who propose an origin from the winds of post-AGB stars and planetary nebula nuclei. Relatively rare are the Group 3 grains, which show slight enrichments in ${ }^{16} \mathrm{O}$. Possible stellar sources include low-metallicity, low-mass AGB stars and SNe. The Group 4 grains make up about $10 \%$ of all grains. These grains exhibit enrichments in ${ }^{18} \mathrm{O}$ and slight to moderate enrichments in ${ }^{17} \mathrm{O}$, except for one olivine grain in which ${ }^{17} \mathrm{O}$ is strongly depleted [36]. The favoured formation sites of the Group 4 grains are SNII ejecta (see section 3). Only two grains have been found with strong enrichments in ${ }^{16} \mathrm{O}$ (see also section 3), the expected predominant signature of SN grains [37]. A few grains show very strong enrichments in ${ }^{17} \mathrm{O}$, possibly pointing to nova origins [32, 38].

Many but not all presolar oxide and silicate grains carry radiogenic ${ }^{26} \mathrm{Mg}$. Inferred ${ }^{26} \mathrm{Al} /{ }^{27} \mathrm{Al}$ ratios are typically $10^{-4}$ to $10^{-2}$ for Group 1 , 3, and 4 grains; Group 2 grains tend to have higher ${ }^{26} \mathrm{Al} /{ }^{27} \mathrm{Al}$ ratios from $10^{-3}$ to 0.2 , which may be the result of CBP [32]. The Siisotopic compositions of presolar silicates plot along the SiC MS line [39-40]. This observation confirms the GCE interpretation of the SiC MS line because O-rich grains form early in the evolution of AGB stars when no or only little dredge-up of s-process Si may have occurred. 


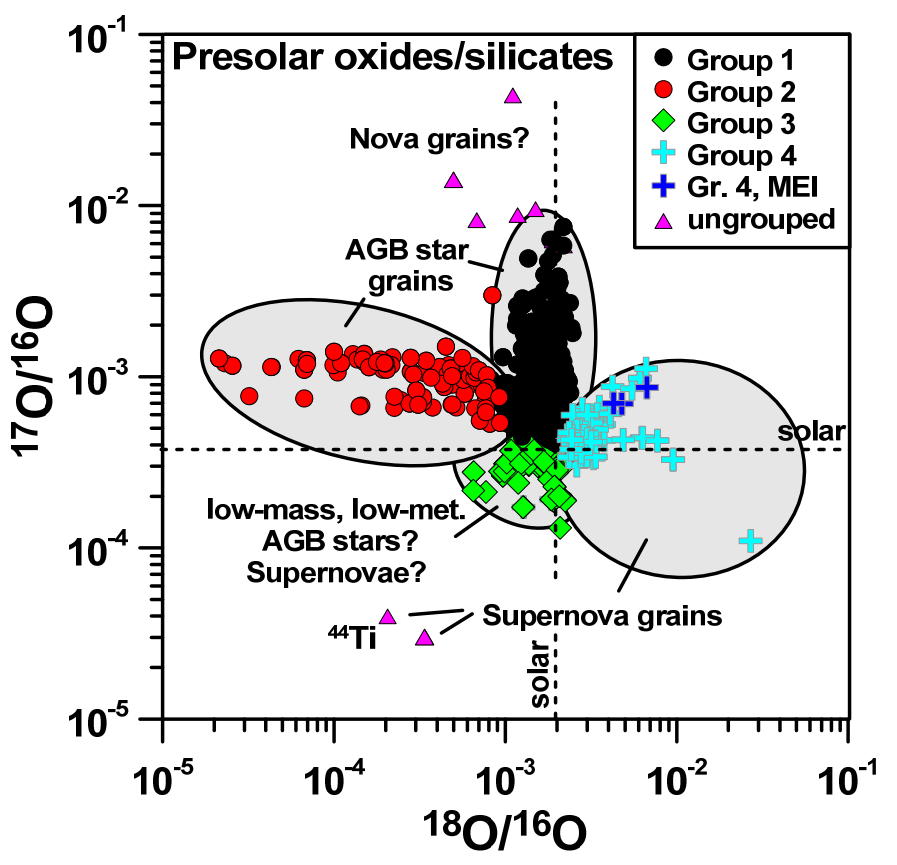

Figure 4. Oxygen-isotopic compositions of the different groups of presolar oxide and silicate grains. The solar ratios are indicated by the dashed lines. "Gr. 4, MEI”: Group 4 grains, multi-element isotope data; Data sources: [32] for Gr. 4, MEI, [38] for the ${ }^{44}$ Ti-rich grain; for the other grains $[32,39,41]$ and references in [3-5].

\section{Recent advances}

In the following I will discuss selected findings in recent years which have advanced our understanding of presolar grains from $\mathrm{SNe}$ and which gave new insights into SN nucleosynthesis, chemistry, and dust formation.

1. Until recently only one $\mu \mathrm{m}$-sized $\mathrm{SiC}$ grain with strong enrichments in ${ }^{29} \mathrm{Si}$ and ${ }^{30} \mathrm{Si}$ has been found. With the NanoSIMS it became possible to extend the isotope studies to submicrometer-sized grains and this revealed several SiC grains with very heavy $\mathrm{Si}$ as well as two grains with large enrichments in ${ }^{29} \mathrm{Si}$ and depletions in ${ }^{30} \mathrm{Si}$; these grains have been named "unusual” or "Type C” (Fig. 3) [16-19, 29-30]. The Si in these grains is clearly distinct from that in AGB star and nova grains. It is also distinct from that in the SN X grains; however, a large range of Si-isotopic compositions is possible in SNII ejecta if matter from different layers is mixed and a SNII origin is clearly favoured. Heavy Si is found in the O-rich zones (Fig. 5) and, provided sufficient Si from these zones is mixed to the sites in the ejecta where $\mathrm{SiC}$ grains form, it is possible to account for the observed Siisotopic signatures.

2. One of the unusual/Type $\mathrm{C}$ grains has a very high ${ }^{29} \mathrm{Si} /{ }^{30} \mathrm{Si}$ ratio of two times the solar ratio (grain "B" in Fig. 3). This implies relative large contributions from the $\mathrm{O} / \mathrm{Ne}$ zone. It was originally proposed by [42] that the ${ }^{29} \mathrm{Si}$ yield in the $\mathrm{O} / \mathrm{Si}$ and $\mathrm{O} / \mathrm{Ne}$ zones is two times higher than currently predicted and only with this adjustment to SN models it is possible to simultaneously account for the $\mathrm{C}$ - and $\mathrm{Si}$-isotopic ratios as well as the ${ }^{44} \mathrm{Ti} /{ }^{48} \mathrm{Ti}$ in grain " $\mathrm{B}$ " [18]. A twofold increase of the ${ }^{29}$ Si yield can be achieved by a $3 \mathrm{x}$ higher ${ }^{26} \mathrm{Mg}(\alpha, \mathrm{n}){ }^{29} \mathrm{Si}$ rate, which is within the uncertainties of the currently used rate. This has also implications for GCE models which predict way too little (i.e, a factor of 2) ${ }^{29} \mathrm{Si}[11]$. With the modified ${ }^{29} \mathrm{Si}$ yield the discrepancy between predicted and observed ${ }^{29} \mathrm{Si}$ can be largely resolved [18]. 
3. SiC $X$ grains contain up to $0.5 \mathrm{wt} \% \mathrm{Fe}$. Most $\mathrm{X}$ grains exhibit enrichments in ${ }^{57} \mathrm{Fe}$ with ${ }^{57} \mathrm{Fe} /{ }^{56} \mathrm{Fe}$ of up to $2 \mathrm{x}$ the solar ratio; in contrast, ${ }^{54} \mathrm{Fe} /{ }^{56} \mathrm{Fe}$ ratios are essentially normal [43]. It is possible to simultaneously explain the ${ }^{57} \mathrm{Fe} /{ }^{56} \mathrm{Fe}$ and Si-isotopic ratios of $\mathrm{X}$ grains by mixing SNII matter from the He/N, He/C, and Si/S zones (Fig. 5). However, the normal ${ }^{54} \mathrm{Fe} /{ }^{56} \mathrm{Fe}$ is puzzeling since the $\mathrm{Si} / \mathrm{S}$ zone is very rich in ${ }^{54} \mathrm{Fe}$ and one would expect to find enhanced ${ }^{54} \mathrm{Fe}$ in $\mathrm{X}$ grains. It is possible to overcome this problem if Fe from the outer $\mathrm{He} / \mathrm{C}$ and $\mathrm{He} / \mathrm{N}$ zones would have been preferentially trapped, e.g., due to element fractionation between matter from different zones by molecule chemistry. This idea is also supported by the recent finding of $\mathrm{S}$ isotope anomalies (depletions of the heavy isotopes) in two SiC grains with strong enrichments in the heavy $\mathrm{Si}$ isotopes [17-18].

4. The origin of the Group 4 oxide/silicate grains was debated for a long time and among the proposed stellar sources were SNeII and high-metallicity AGB stars. From multi-element isotope data for three Group 4 oxide grains (cf. Fig. 4) the SNII origin is now clearly favoured [32]. This is based on the excellent agreement between the $\mathrm{O}, \mathrm{Mg}$, and/or $\mathrm{Ca}$ isotope data and predictions from $15 \mathrm{M}_{\odot}$ SNII mixing models. In these models most matter comes from the $\mathrm{H}$ and $\mathrm{He} / \mathrm{N}$ zones and the ${ }^{18} \mathrm{O}$ enrichments are the result of admixture of matter from the He/C zone (Fig. 5). Additional support for a SNII origin comes from $\mathrm{Mg}$ and Si isotope data of Group 4 silicate grains [40, 44].

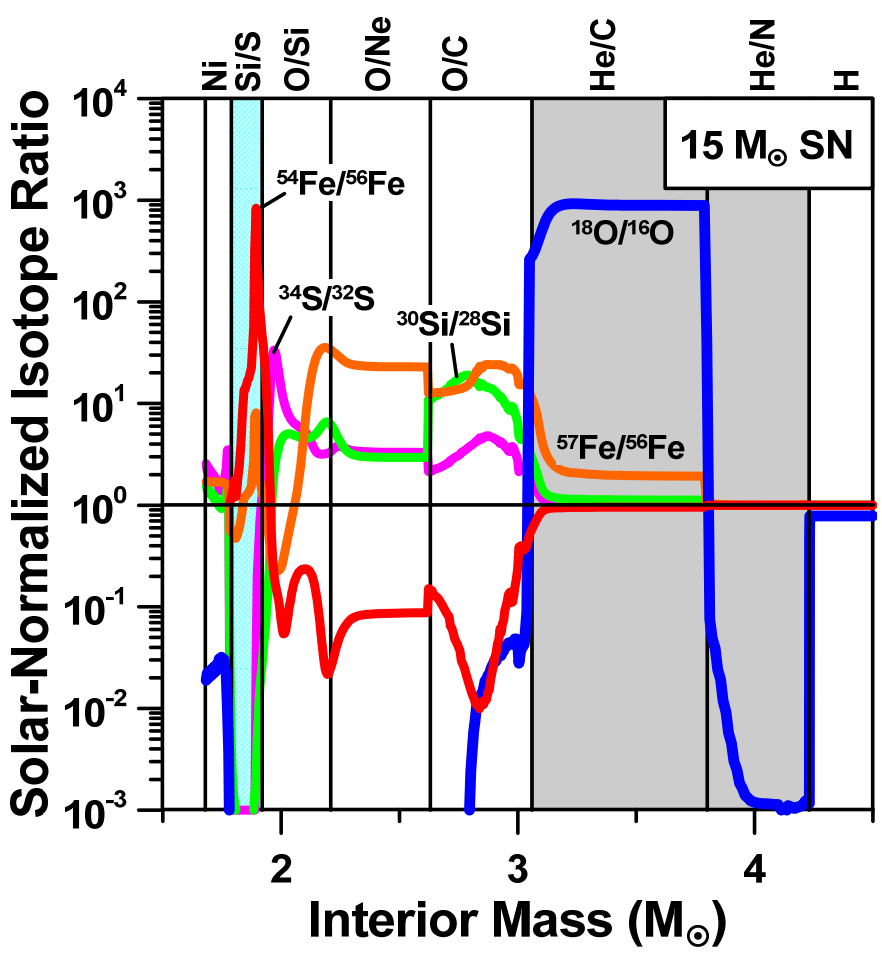

Figure 5. Profiles of solarnormalized $\quad{ }^{18} \mathrm{O} /{ }^{16} \mathrm{O}, \quad{ }^{30} \mathrm{Si} /{ }^{28} \mathrm{Si}$, ${ }^{34} \mathrm{~S} /{ }^{32} \mathrm{~S}, \quad{ }^{54} \mathrm{Fe} /{ }^{56} \mathrm{Fe}$, and ${ }^{57} \mathrm{Fe} /{ }^{56} \mathrm{Fe}$ ratios in the interior of a $15 \mathrm{M}_{\odot}$ SNII [45]. The different SN layers are indicated at the top.

5. The presence of ${ }^{44} \mathrm{Ti}$ at the time of grain formation in presolar $\mathrm{SiC} X$ and low-density graphite grains has been taken as final proof for a SN origin of these grains. Clear evidence for ${ }^{44} \mathrm{Ti}$, based on a ${ }^{44} \mathrm{Ca} /{ }^{40} \mathrm{Ca}$ ratio of $\sim 60 \mathrm{x}$ solar, has now also been found for an extremely ${ }^{16} \mathrm{O}$-enriched spinel grain (Fig. 4) [38]. This finding strongly supports the proposed SN origin of the very rare grains with ${ }^{16} \mathrm{O}$ enrichments. Its $\mathrm{O}$ - and Mg-isotopic compositions as well as its ${ }^{44} \mathrm{Ti} /{ }^{48} \mathrm{Ti}$ ratio can be well explained in the context of the $15 \mathrm{M}_{\odot}$ model of [46]. 
6. It is known since long that meteorites show ${ }^{54} \mathrm{Cr}$ variations on a macroscopic scale and it was speculated that these variations are caused by different amounts of $\mathrm{Cr}$-bearing presolar grains in planetary matter. Just recently, $<200 \mathrm{~nm}$-sized oxide grains with large ${ }^{54} \mathrm{Cr}$ enrichments have been found [47-48]. The most likely sources of these grains are SNeII, in particular the $\mathrm{O} / \mathrm{Ne}$ and $\mathrm{O} / \mathrm{C}$ zones (see also the contribution by Nittler et al. in this issue).

\section{Unsolved problems}

Many of the isotopic properties of presolar grains can be explained, at least qualitatively, by stellar models. The presolar grain data have been used to constrain stellar nucleosynthesis models and mixing conditions in SN ejecta, which has openened a new window to astrophysics. Nevertheless, there are still numerous unsolved problems, e.g., the following:

1. AGB star grains appear to come largely from stars with masses between 1.2 and $3 \mathrm{M}_{\odot}$. However, AGB stars heavier than $4 \mathrm{M}_{\odot}$ are expected to contribute significantly to the interstellar dust inventory [49] which is at odds with the inferences from the presolar grain data.

2. Presolar oxide/silicate grains from AGB stars tend to have higher ${ }^{26} \mathrm{Al} /{ }^{27} \mathrm{Al}$ ratios than $\mathrm{SiC}$ grains [50]. This is surprising in view of the fact that the parent stars of SiC follow the parent stars of O-rich dust in their evolutionary sequence. Cool bottom processing is required to account for the high ${ }^{26} \mathrm{Al} /{ }^{27} \mathrm{Al}$ ratios of O-rich grains but not for the Al-isotopic ratios in SiC from AGB stars. A better understanding of the role of CBP in the evolution of low- and intermediate-mass stars may help to shed more light on this issue.

3. Why are there only so few $\mathrm{SN}$ grains with large ${ }^{16} \mathrm{O}$ excesses? The intermediate O-rich zones in SNII are extremely ${ }^{16} \mathrm{O}$-rich and one would expect to find much more of these grains than $\mathrm{SN}$ grains with ${ }^{18} \mathrm{O}$ excesses.

4. The comparison of SN grain data with model predictions is based on ad-hoc mixing calculations. More realistic models that consider the physics of mixing and molecule chemistry are clearly needed.

Acknowledgements - I wish to thank the organizers of the Nuclei in the Cosmos XI conference for the invitation to present this paper.

\section{References}

[1] T. Bernatowicz et al., Evidence for interstellar SiC in the Murray carbonaceous meteorite, Nature 330 (1987) 728.

[2] R. S. Lewis, M. Tang, J. F. Wacker, E. Anders, E. Steel, Interstellar diamonds in meteorites, Nature 326 (1987) 160.

[3] P. Hoppe, Reservoir for Comet Material: Circumstellar Grains, Space Sci. Rev. 138 (2008) 43.

[4] K. Lodders, S. Amari, Presolar grains from meteorites: Remnants from the early times of the solar system, Chemie der Erde 65 (2005) 93.

[5] E. Zinner, Presolar Grains, in Meteorites, Comets, and Planets, Elsevier, Amsterdam 2007.

[6] P. Hoppe, S. Amari, E. Zinner, T. Ireland, R. S. Lewis, Carbon, nitrogen, magnesium, silicon and titanium isotopic compositions of single interstellar silicon carbide grains from the Murchison carbonaceous chondrite, ApJ 430 (1994) 870. 
[7] M. Lugaro et al., Isotopic compositions of strontium, zirconium, molybdenum, and barium in single presolar SiC grains and asymptotic giant branch stars, ApJ 593 (2003) 486.

[8] P. W. Merrill, Spectroscopic observations of stars of class S, ApJ 116 (1952) 21.

[9] M. R. Savina et al., Extinct technetium in presolar silicon carbide grains, Science 303 (2004) 649.

[10] M. Lugaro, E. Zinner, R. Gallino, S. Amari, Si isotopic ratios in mainstream presolar SiC grains revisited, ApJ 527 (1999) 369.

[11] F. X. Timmes, D. D. Clayton, Galactic evolution of silicon isotopes: Application to presolar SiC grains from meteorites, ApJ 472 (1996) 723.

[12] E. Zinner et al., Silicon and carbon isotopic ratios in AGB stars: SiC grain data, models, and the galactic evolution of the Si isotopes, ApJ 650 (2006) 350.

[13] S. Amari et al., Presolar SiC grains of type Y: Origin from low-metallicity AGB stars, ApJ 546 (2001) 248.

[14] P. Hoppe et al., Meteoritic silicon carbide grains with unusual Si-isotopic compositions: Evidence for an origin in low-mass metallicity asymptotic giant branch stars, ApJ 487 (1997) L101.

[15] S. Amari, L. R. Nittler, E. Zinner, K. Lodders, R. S. Lewis, Presolar SiC grains of type A and B: Their isotopic compositions and stellar origins, ApJ 559 (2001) 463.

[16] S. Amari, E. Zinner, R. S. Lewis, A singular presolar SiC grain with extreme ${ }^{29,30}$ Si excesses, ApJ, 517 (1999) L59.

[17] F. Gyngard, L. R. Nittler, E. Zinner, Presolar SiC of Type C, Met. Planet. Sci. 45 (2010) A72.

[18] P. Hoppe et al., NanoSIMS studies of small presolar SiC grains: New insights into supernova nucleosynthesis, chemistry, and dust formation, ApJ 719 (2010) 1370.

[19] E. Zinner, F. Gyngard, L. R. Nittler, Automated C and Si isotopic analysis of presolar SiC grains from the Indarch enstatite chondrite, Lunar Planet. Sci. 41 (2010) abstract \#1359.

[20] P. Hoppe, R. Strebel, P. Eberhardt, S. Amari, R. S. Lewis, Isotopic properties of silicon carbide X grains from the Murchison meteorite in the size range 0.5-1.5 $\mu \mathrm{m}$, MAPS 35 (2000) 1157.

[21] P. Hoppe, A. Besmehn, Evidence for extinct Vanadium-49 in presolar silicon carbide grains from supernovae, ApJ 576 (2002) L69.

[22] Y. Lin, F. Gyngard, E. Zinner, Isotopic analysis of supernova SiC and $\mathrm{Si}_{3} \mathrm{~N}_{4}$ grains from the Qingzhen (EH3) chondrite, ApJ 709 (2010) 1157.

[23] L. R. Nittler, S. Amari, E. Zinner, S. E. Woosley, R. S. Lewis, Extinct ${ }^{44}$ Ti in presolar graphite and SiC: Proof of a supernova origin, ApJ 462 (1996) L31.

[24] B. S. Meyer, D. D. Clayton, L.-S. The, Molybdenum and zirconium isotopes from a supernova neutron burst, ApJ 540 (2000) L49.

[25] L. R. Nittler et al., Silicon nitride from supernovae, ApJ 453 (1995) L25.

[26] S. Amari, X. Gao, L. R. Nittler, E. Zinner, Presolar grains from novae, ApJ 551 (2001) 1065.

[27] L. R. Nittler, P. Hoppe, Are presolar silicon carbide grains from novae actually from supernovae?, ApJ 631 (2005) L89.

[28] S. Messenger, L. P. Keller, F. Stadermann, R. M. Walker, E. Zinner, Samples of stars beyond the solar system: Silicate grains in interplanetary dust, Science 300 (2003) 105.

[29] T. K. Croat, T. J. Bernatowicz, Unusual 29,20Si-rich SiCs of massive star origin found within graphites from the Murchison meteorite, Astron. J. 139 (2010) 2159.

[30] MPI Mainz, unpublished (2010) 
[31] L. R. Nittler, C. M. O. D. Alexander, X. Gao, R. M. Walker, E. Zinner, Stellar sapphires: The properties and origins of presolar $\mathrm{Al}_{2} \mathrm{O}_{3}$ in meteorites, ApJ 483 (1997) 475.

[32] L. R. Nittler et al., Aluminum-, calcium- and titanium-rich oxide stardust in ordinary chondrite meteorites, ApJ 682 (2008) 1450.

[33] L. R. Nittler, On the mass and metallicity distributions of the parent AGB stars of O-rich presolar dust, PASA 26 (2009) 271.

[34] K. M. Nollett, M. Busso, G. J. Wasserburg, Cool bottom processes on the thermally pulsing asymptotic giant branch and the isotopic composition of circumstellar dust grains, ApJ $5 \mathbf{8 2}$ (2003) 1036.

[35] J. Buntain et al., Wind beyond the tip of the AGB and its relevance to stardust grain data, Proc. of Science, this issue.

[36] S. Messenger, L. P. Keller, D. S. Lauretta, Supernova Olivine from Cometary Dust, Science 309 (2005) 737.

[37] L. R. Nittler, C. M. O. D. Alexander, J. Wang, X. Gao, Meteoritic oxide grain from supernova found, Nature 393 (1998) 222.

[38] F. Gyngard, E. Zinner, L. R. Nittler, A. Morgand, F. J. Stadermann, K. M. Heynes, Automated NanoSIMS measurements of spinel stardust from the Murray meteorite, ApJ 717 (2010) 107.

[39] A. N. Nguyen et al., Characterization of presolar silicate and oxide grains in primitive carbonaceous chondrites, ApJ 656 (2007) 1223.

[40] C. Vollmer, P. Hoppe, F. Brenker, Si-isotopic compositions of presolar silicate grains from red giant stars and supernovae, ApJ 684 (2008) 611.

[41] C. Vollmer, P. Hoppe, F. J. Stadermann, C. Floss, F. Brenker, NanoSIMS analysis and Auger electron spectroscopy of silicate and oxide stardust from the carbonaceous chondrite Acfer 094, Geochim. Cosmochim. Acta 73 (2009) 7127.

[42] C. Travaglio, R. Gallino, E. Zinner, S. Amari, S. Woosley, in Nuclei in the Cosmos V, Editions Frontieres, Paris 1998.

[43] K. K. Marhas, S. Amari, F. Gyngard, E. Zinner, R. Gallino, Iron and nickel isotopic ratios in presolar SiC grains, ApJ 689 (2008) 622.

[44] A. Nguyen, S. Messenger, M. Ito, Z. Rahman, Mg isotopic measurement of FIB-isolated presolar silicate grains, Lunar Planet. Sci. 41 (2010) abstract \#2413.

[45] T. Rauscher, A. Heger, R. D. Hoffman, S. E. Woosley, Nucleosynthesis in massive stars with improved nuclear and stellar physics, ApJ 576 (2002) 323.

[46] S. E. Woosley, A. Heger, Nucleosynthesis and remnants in massive stars of solar metallicity, Phys. Rep. 442 (2007) 269.

[47] N. Dauphas et al., Neutron-rich chromium isotope anomalies in supernova nanoparticles, ApJ 720 (2010) 1577.

[48] L. R. Nittler et al., Extreme uncorrelated 54Cr, 17O, and 180 enrichments in sub-micron Orgueil grains, Lunar Planet. Sci. 41 (2010) abstract \#2071.

[49] H.-P. Gail, S. V. Zhukovska, P. Hoppe, M. Trieloff, Stardust from asymptotic giant branch stars, ApJ 698 (2009) 1136.

[50] E. Zinner et al., NanoSIMS isotopic analysis of small presolar grains: Search for $\mathrm{Si}_{3} \mathrm{~N}_{4}$ grains from AGB stars and Al and Ti isotopic compositions of rare presolar SiC grains, GCA 71 (2007) 4786. 